\title{
Nonhierarchical Dispersal Promotes Stability and Resilience in a Tritrophic Metacommunity
}

\author{
Eric J. Pedersen, ${ }^{1, \star}$ Justin N. Marleau, ${ }^{2}$ Monica Granados, ${ }^{2}$ Holly V. Moeller, ${ }^{3}$ \\ and Frédéric Guichard ${ }^{2}$
}

1. Center for Limnology, University of Wisconsin-Madison, Madison, Wisconsin 53706; 2. Department of Biology, McGill University, 1205 Docteur Penfield, Montreal, Quebec H3A 1B1, Canada; 3. Biology Department, Stanford University, Stanford, California 94305

Submitted October 2, 2014; Accepted October 23, 2015; Electronically published March 16, 2016

Online enhancements: appendixes.

\begin{abstract}
Community interactions (e.g., predation, competition) can be characterized by two factors: their strengths and how they are structured between and within species. Both factors play a role in determining community dynamics. In addition to trophic interactions, dispersal acts as an interaction between separate populations. As with other interactions, the structure of dispersal can affect the stability of a system. However, the primary structure that has been studied in consumer-resource models has been hierarchical dispersal, where between-patch dispersal rates increase with trophic level. Here we use analytical, numerical, and simulation approaches on a two-patch, three-species metacommunity model to investigate the relationship between structure and community stability and resilience. We show that metacommunity stability is greater in systems with both weak and strong dispersal rates. Our system is stabilized by the formation of patterns when predators disperse frequently and herbivores disperse rarely, and via asynchrony when both predators and herbivores disperse infrequently. Our results show how interaction strengths within both trophic and spatial networks shape metacommunity stability.
\end{abstract}

Keywords: metacommunity, movement ecology, interaction strength, food webs, stability, ecological resilience.

\section{Introduction}

Ecologists have typically treated dispersal and trophic interactions as two separate interacting forces determining community stability. Researchers studying the stability of consumer-resource networks have focused on the structural properties of networks of species interactions, such as the pattern of positive and negative interactions in food webs (e.g., Levins 1974; Justus 2006), the number and strength of food web connections (e.g., May 1972, 1973), or the frequency of specific types of ecological interactions, such as

* Corresponding author; e-mail: eric.pedersen@wisc.edu.

Am. Nat. 2016. Vol. 187, pp. E116-E128. (C) 2016 by The University of Chicago. 0003-0147/2016/18705-55795\$15.00. All rights reserved. DOI: $10.1086 / 685773$ predation or competition (e.g., Allesina and Tang 2012). In comparison, ecologists studying the stability of spatial communities connected by dispersal have focused on the effects of either varying interpatch dispersal rates of all species together (e.g., Pillai et al. 2011) or varying the structure of connectivity in landscapes (Fagan 2002). These separate approaches effectively treat dispersal as a community-level property, distinct from species-specific interactions such as feeding, mating, or facilitation. However, there is no reason this should be so; dispersal is simply an interaction between spatially separated groups of a single species.

Numerous studies have focused on how interaction strengths are structured across networks of ecological agents and how these structural properties affect the stability of ecosystem processes. Analyses of consumer-resource interactions within food webs reveal consistent structural features. For instance, dissected food webs are dominated by many weak interactions and a few strong interactions (Paine 1992; Wootton 1997; McCann et al. 1998; Neutel et al. 2002), with strong interactions occurring at basal trophic levels (de Ruiter et al. 1995).

As with trophic interactions, dispersal traits tend to be highly structured within communities. Perhaps the bestknown pattern is the hierarchy of dispersal: both movement rates (McCann et al. 2005) and the body size-home range scaling relationship (Haskell et al. 2002) tend to increase with trophic level. However, faster movement rates at small scales may not carry over into more frequent interpatch dispersal at larger scales. For instance, between-lake dispersal of plankton and fish tends to be antihierarchical (lowertrophic-level organisms dispersing more frequently than higher-trophic-level ones), as lighter organisms tend to get dispersed more readily by animals and physical factors (Beisner et al. 2006). Understanding how the structure of dispersal varies at large scales and how these structures act to change the stability of community dynamics may help explain the macroecological patterns of stable and cyclic pat- 
terns in field data (Kendall et al. 1998). Further, changes in species dispersal rates may have been one factor stabilizing previously oscillating populations (Ims et al. 2008).

The population-dynamic consequences of the vertical structure of dispersal rates have been examined in the literature on pattern formation in predator-prey systems, first discussed by Segel and Jackson (1972). This body of work has focused less on how dispersal affects ecosystem stability in the broad sense used in community ecology (Loreau et al. 2002). It has instead focused on which combinations of dispersal rates for predator and prey can give rise to spatially inhomogeneous patterns in bitrophic systems. However, pattern formation can be seen as stabilizing if the local patches fluctuate in the absence of dispersal: if a spatially homogeneous state is oscillatory, the new patterned state may be less temporally variable, as a result of either the patterns being fixed in space or asynchronous oscillations between distant sites reducing average global variability (Guichard and Gouhier 2014).

Research on pattern formation has concluded that the vertical structure of dispersal can lead to new spatially heterogeneous states if three criteria are satisfied (Turing 1952; Segel and Jackson 1972): (1) one of the species acts as an activator, in that its rate of growth increases with an increase in its own population density; (2) the second species acts as an inhibitor, in that its rate of growth decreases with an increase in its own density; and (3) the inhibitor species disperses more frequently (or interacts over longer spatial scales) than the activator species (Rietkerk and van de Koppel 2008; Della Rossa et al. 2012). In terms of interaction strengths, this means that the more frequently dispersing species has to interact negatively with itself and the less frequently dispersing species has to interact positively.

In most of the original literature, the predator was treated as the inhibitor and the prey as the activator. However, early models had to assume prey Allee effects and predator selfinhibition to meet this assumption (Alonso et al. 2002; Fasani and Rinaldi 2011). In fact, many mechanisms that allow spatially inhomogeneous dynamics also result in the predator acting as an activator and the prey acting as an inhibitor. In those cases, hierarchically structured dispersal rates would actually act to suppress spatial patterns. More complex models have suggested that spatial coupling at other trophic levels or even with nutrients can lead to spatial patterning (Koelle and Vandermeer 2005; Marleau et al. 2010, 2014), as adding additional species may shift which species are acting as activators or inhibitors. In systems with more than two species, the activator-inhibitor paradigm can be generalized to talk about sets of activators and inhibitors (Della Rossa et al. 2012), but this still leaves the question, where in a food web do we expect to find activators or inhibitors, and therefore which dispersal structures can act to spatially stabilize ecosystems?
It is also possible for complex spatial dynamics to appear in consumer-resource systems even when both species have the same dispersal rates, because of the interaction of infrequent dispersal and local oscillatory dynamics driven by ecological interactions, resulting in asynchrony (e.g., Pascual 1993; Gouhier et al. 2010). However, there has been very little research on how altering the structure of dispersal rates, rather than simply scaling all dispersal rates, alters the synchrony of metacommunities. Further, little is known about the conditions under which each of the two stabilizing mechanisms dominates: which dispersal structures result in spatial synchrony, asynchrony, or patterns?

We use a two-patch tritrophic model to demonstrate how the vertical structure of dispersal rates and within-patch intraspecific interaction strength combine to determine the temporal variability of the community and the resilience of alternate dynamical regimes. We combine analytical methods and numerical analysis of the possible dynamic states in this system to determine whether the system exhibits spatially patterned or unpatterned dynamics under different combinations of trophic dispersal structures and resource self-regulation strengths. We show that stability and resilience of this metacommunity both increase as dispersal rates become more nonhierarchical: when resource self-regulation is weak, this system will stabilize only when the lowest trophic level disperses frequently and the intermediate trophic level disperses rarely. Further, we demonstrate that this system is ecologically resilient when the strength of resource selfregulation is sufficiently strong.

\section{Material and Methods}

\section{Model Formulation}

Our analysis is based around a two-patch community with three-species food chains, coupled by diffusive dispersal. State variables and model parameters are measured in units of biomass and biomass flux per unit time (see app. B; apps. A-C available online). We define one of the species to be the basal resource $(R)$. The basal resource is consumed by herbivores $(H)$ that are in turn consumed by predators $(P)$. Predator growth rates and mortality-plus-respiration are both linearly dependent on predator density. All three species disperse between communities at their own densityindependent per capita rates. The two communities can then be described by the following system of differential equations:

$$
\begin{aligned}
& \frac{\mathbb{d} R_{i}}{\mathbb{d} t}=F_{\mathrm{R}}\left(R_{i}, H_{i}\right)+d_{\mathrm{R}} \cdot\left(R_{j}-R_{i}\right), \\
& \frac{\mathbb{d} H_{i}}{\mathbb{d} t}=F_{\mathrm{H}}\left(R_{i}, H_{i}, P_{i}\right)+d_{\mathrm{H}} \cdot\left(H_{j}-H_{i}\right), \\
& \frac{\mathbb{d} P_{i}}{\mathbb{d} t}=F_{\mathrm{P}}\left(H_{i}\right) \cdot P_{i}+d_{\mathrm{P}} \cdot\left(P_{j}-P_{i}\right),
\end{aligned}
$$


where $i, j \in\{1,2\}$ and $i \neq j$. The functions $F_{\mathrm{R}}, F_{\mathrm{H}}$, and $F_{\mathrm{P}}$ encompass all within-patch interactions between the trophic levels (see eq. [4] for the functional forms of these equations used in our numerical analyses). The terms $d_{\mathrm{R}}$, $d_{\mathrm{H}}$, and $d_{\mathrm{P}}$ represent density-independent dispersal rates between patches $i$ and $j$.

While tritrophic models exhibit a wide range of dynamical behavior (van Voorn et al. 2010), we focus our analysis principally on how different structural relationships between dispersal rates can change the predictions derived from well-mixed communities. We compare how several qualitative measures of ecosystem stability shift when we vary both the strength of local feedbacks and the structural pattern of dispersal rates. To do so, we examine our model by using local stability analysis, bifurcation diagrams, and numerical simulations to get a full measure of the effects of each type of structure. Within this model system, we define hierarchical dispersal as $d_{\mathrm{P}} \geq d_{\mathrm{H}} \geq d_{\mathrm{R}}$. We also identify two other feasible structural relationships that show dynamics qualitatively different from hierarchical dispersal: nonmonotonic dispersal $\left(d_{\mathrm{P}} \geq d_{\mathrm{H}}<d_{\mathrm{R}}\right)$ and antihierarchical dispersal $\left(d_{\mathrm{P}}<d_{\mathrm{H}}<d_{\mathrm{R}}\right)$

\section{Stability Criteria}

When we refer to potential stabilizing effects of various dispersal structures, we consider three different possible forms of stability (Loreau et al. 2002). The first is qualitative stability, that is, when a system is on an attractor, the speed at which the system returns to that trajectory after a small perturbation or whether the system has a tendency to return to it at all. We obtained information about stable and unstable regions in parameter space by tracking bifurcation points where different attractors gained or lost local stability.

The second is variability: how variable population dynamics are over time, relative to their long-run average level. This will depend on the scale considered. Each patch may be oscillating, but average densities across the whole metacommunity may not be varying at all if the two patches are oscillating in antiphase. We looked for qualitative changes in variability by determining what changes in parameter values or initial conditions caused the system to switch from both patches oscillating synchronously to either an asymmetric equilibrium or the patches oscillating out of phase.

The third is ecological resilience: the amount of disturbance a system can withstand before switching into an alternate regime. This includes resistance to extinction or invasion, as these are by definition changes in the state of a system (Gunderson 2000). We quantified this by determining which parameter combinations lead to bistable alternate stable states. For parameter combinations that allowed alternate stable states, we determined the size of the basin of attraction for each alternate stable state, as the volume of an attractor's basin can act as an indicator of how stable the attractor is to larger perturbations (Menck et al. 2013). Parameter combinations with alternate stable states were considered to be less resilient than ones with only a single stable state, and the state with the larger basin was considered more resilient to disturbance.

\section{Equilibrium Analysis}

Differences in dispersal by themselves cannot shift a system from a symmetric equilibrium to symmetric oscillations. These shifts occur because of changes in within-patch interaction strengths. In the framework defined in the previous section, shifting from a symmetric equilibrium to some form of spatial pattern results primarily in a change in qualitative stability and does not necessarily change a system's resilience or variability. However, it is still useful to understand what dispersal structures lead a symmetric equilibrium to shift to a spatially heterogeneous attractor. Moreover, insights from the equilibrium case often carry over to predicting the effect of that structure on nonequilibrium dynamics.

We used a theorem developed by Jansen and Lloyd (2000) to determine local stability of spatially homogeneous solutions in multipatch systems. In our case, each patch was a community, and the spatially homogeneous solutions were equilibria (Jansen and Lloyd 2000). In a system with $k$ species and $n$ patches (communities), the theorem requires defining both a dispersal matrix (D), which is a $k \times k$ matrix, and a connectivity matrix $(\mathbf{C})$, which is an $n \times n$ matrix, using connectivity in the sense defined by Jansen and Lloyd (2000). The connectivity matrix describes the topology of the landscape (i.e., which patch is connected to which). The dispersal matrix is a diagonal matrix, with entries that describe the rates at which each individual of a given species will disperse from one patch to the other, given that they are connected. The dispersal and connectivity matrices associated with equation (1) can be found in the description of the more general model (app. A).

With both matrices defined and having certain properties that are met here (see Jansen and Lloyd 2000 for more details), the spatially homogeneous solution is locally stable if the eigenvalues for the following matrices all have negative real parts:

$$
\mathbf{V}_{i}=\mathbf{J}+\lambda_{i} \mathbf{D}
$$

Here, $\mathbf{J}$ is the Jacobian matrix for a single community at the homogeneous equilibrium and $\lambda_{i}$ is the $i$ th eigenvalue of the connectivity matrix. The individual entries of $\mathbf{J}, j_{A B}$, represent how species $B$ affects species $A$ 's growth rate at equilibrium. The eigenvalues of the connectivity matrix (specified in app. A, eq. [A2]) are $\lambda_{1}=0$ and $\lambda_{2}=-2$. Therefore, we have the following two expressions for the $\mathbf{V}_{i}$ matrices: 


$$
\begin{aligned}
& \mathbf{V}_{1}=\mathbf{J}, \\
& \mathbf{V}_{2}=\mathbf{J}-2 \mathbf{D} .
\end{aligned}
$$

Because we were interested in departures from a locally stable spatially homogeneous solution, we considered only the eigenvalues of $\mathbf{V}_{2}$. While direct computation of these eigenvalues is possible, we used the Routh-Hurwitz conditions to determine the signs of the eigenvalues (see app. A and May 1973 for a description of this approach). These conditions were then reformulated to derive critical dispersal rates of the different species that cause the destabilization of the spatially homogeneous solution (Marleau et al. 2010), which in biological terms means that spatial heterogeneity in species densities is created when dispersal rates are higher than their critical values.

\section{Nonequilibrium Analysis}

Our equilibrium analysis showed which combinations of dispersal parameters lead to the emergence of different spatial solutions from a spatially homogeneous equilibrium and thus what dynamics we might expect under different dispersal structures. While the local stability analysis of equilibria can be derived from direct analysis of the general equations, following nonequilibrium attractors requires the use of numerical bifurcation techniques. To do so, we used AUTO-07p (Doedel et al. 2010), software dedicated to the numerical analysis of nonlinear dynamical systems, which is able to track bifurcations in nonequilibrium systems. We also used AUTO-07p to find regions of parameter space where two stable attractors coexisted (bistability).

Dispersal structure may stabilize nonequilibrium metacommunity dynamics in two ways. First, it may generate a stable patterned equilibrium in a system that would be purely oscillatory if well mixed. Second, it may lead to desynchronization of oscillations between patches, leading to a decrease in the variability of aggregate populations. In both cases, dispersal structure may also act to stabilize a system in response to changes in trophic-interaction strength, as the patterned equilibrium or asynchronous oscillatory state may be more stable to parameter changes than the well-mixed system. Further, dispersal structure may change the ecological resilience, compared to that of a well-mixed system, by creating alternate stable states or destabilizing a previously existing alternate stable state. None of these shifts in stability are observable by simply looking at the local stability of a well-mixed equilibrium. They can be detected only by following each type of attractor through changes in parameter space.

We used a specific parameterization of equation (1), assuming logistic dynamics for $R_{i}$, a type I interaction be- tween $R_{i}$ and $H_{i}$, a type II interaction between $H_{i}$ and $P_{i}$, and density-independent mortality for $H$ and $P$ :

$$
\begin{aligned}
\frac{\mathbb{d} R_{i}}{\mathbb{d} t}= & R_{i}-\beta R_{i}^{2}-0.1 R_{i} H_{i}+d_{\mathrm{R}}\left(R_{j}-R_{i}\right), \\
\frac{\mathbb{d} H_{i}}{\mathbb{d} t}= & 0.1 R_{i} H_{i}-\frac{0.1 H_{i}}{1+H_{i}} P_{i}-0.04 H_{i} \\
& +d_{\mathrm{H}}\left(H_{j}-H_{i}\right), \\
\frac{\mathbb{d} P_{i}}{\mathbb{d} t}= & \frac{0.1 H_{i}}{1+H_{i}} P_{i}-0.08 P_{i}+d_{\mathrm{P}}\left(P_{j}-P_{i}\right),
\end{aligned}
$$

where $i, j \in\{1,2\}$ and $i \neq j$. Here, $\beta$ is the rate at which self-regulation by the resource reduces its own growth rate and $d_{\mathrm{R}}, d_{\mathrm{H}}$, and $d_{\mathrm{P}}$ are the trophic-level-specific dispersal rates. We used a type I functional response for the herbivore in order to minimize the occurrence of chaotic dynamics within the nonspatial system, which are known to occur with type II functional responses (e.g., Hastings and Powell 1991). We set predator mortality higher than herbivore mortality for the same reason. If we assume that $R_{i}, H_{i}$, and $P_{i}$ are measured in units of kilograms of biomass and rate parameters per week, this model is consistent with a planktoninvertebrate-fish food web (app. B). Assimilation efficiencies in our model were set at 1 . Such high assimilation efficiency leads to faster feedbacks and therefore to a larger range of parameters associated with oscillatory dynamics. However, our results were robust to smaller assimilation efficiencies (app. B).

\section{Analysis of Ecological Resilience}

One of the possible dynamic outcomes for this model is a bistable state, where two or more attractors are locally stable. However, while bifurcation analysis can tell us which attractors are locally stable for a given set of parameters, this does not indicate how many initial conditions lead to a given attractor. Neither analytical techniques nor numerical bifurcation could tell us about the relative volume of attractors, so we used simulations to measure them. All simulations were conducted in $\mathrm{R}$ (ver. 3.0.1; R Core Team 2013).

For each pair of parameters of interest, we constructed a $30 \times 30$ grid of parameter sets by varying the two parameters while holding all others constant. For each parameter set, we generated 400 initial conditions by sampling $R, H$, and $P$ values for each patch from a range of densities from 0 to $\beta^{-1}$ individuals per patch. We chose $\beta^{-1}$ as a maximum initial population because it corresponds to the highest possible resource carrying capacity achievable in the absence of herbivores or predators and thus represents an upper bound 
on the total population density possible in a patch without outside subsidy. We used a Latin hypercube sampling scheme (using the lhs R package; Carnell 2012) to generate initial densities, to ensure that we efficiently sampled initial condition space. The same set of initial conditions (rescaled by $\beta^{-1}$ ) were used for simulations for all parameter values.

For each initial condition for each parameter set, we ran the simulation for 15,000 time units to allow initial transients to settle out. Simulations were performed with the deSolve package (ver. 1.10.8; Soetaert et al. 2010), using the lsoda adaptive solver. After simulation, the last 5,000 time units were retained and split into two time series of 2,500 time units each. A set of statistics were calculated for each time series of herbivore populations $\left(H_{1}\right.$ and $\left.H_{2}\right)$ to determine its attractor. If the ratio of the sums of the variances of herbivore densities to the sum of the average herbivore densities $\left(\left(\operatorname{Var}\left(H_{1}\right)+\operatorname{Var}\left(H_{2}\right)\right) /\left(\operatorname{mean}\left(H_{1}\right)+\operatorname{mean}\left(H_{2}\right)\right)\right)$ was greater than 0.01 , the simulation was classified as oscillating. If the absolute difference between average herbivore densities divided by the maximum observed herbivore density $\left(\left|\operatorname{mean}\left(H_{1}\right)-\operatorname{mean}\left(H_{2}\right)\right| / \max \left(\operatorname{mean}\left(H_{1}\right)\right.\right.$, mean $\left.\left.\left(H_{2}\right)\right)\right)$ was greater than 0.05 , the simulation was classified as asymmetric. If the simulation was not asymmetric and the correlation between the two herbivore densities over time $\left(\left(\operatorname{mean}\left(H_{1} \cdot H_{2}\right)-\operatorname{mean}\left(H_{1}\right) \cdot \operatorname{mean}\left(H_{2}\right)\right) /\left(\operatorname{Var}\left(H_{1}\right)^{0.5}\right.\right.$. $\left.\left.\operatorname{Var}\left(\mathrm{H}_{2}\right)^{0.5}\right)\right)$ was less than 0.95 , it was classified as asynchronous.

We tested for convergence to an attractor by comparing all three test statistics for the two halves of the final time series. Convergence required that differences for all statistics be of the same order as their tolerance values used to characterize attractors. If the time series had not converged, the model was run for an additional 5,000 time units and reevaluated. This process was repeated until all statistics converged.

\section{Results \\ Conditions for Destabilizing the Spatially Homogeneous Equilibria}

We first examined the general case: three trophic levels in two patches connected by diffusive dispersal, with within-patch dynamics governed by any dynamics described by equation (1). We derived conditions for transitions from the spatially symmetric three-species equilibrium due to changes in dispersal rates (app. A). The key result from the derivation is that both kinds of transitions that can occur to destabilize the three-species coexistence equilibrium are possible in our system: the transition to spatially asymmetric equilibria and the transition to asynchronous oscillations.

From the Routh-Hurwitz conditions, we derived two critical dispersal levels that determine the transitions to spatially heterogeneous equilibria and to asynchronous oscillations from the symmetric equilibrium (app. A). The critical value of the predator dispersal rate needed to transition into asymmetric equilibria is

$$
d_{\mathrm{P}, \mathrm{c}}=\frac{1}{2} \frac{\left(j_{\mathrm{RR}}-2 d_{\mathrm{R}}\right) j_{\mathrm{HP}} j_{\mathrm{PH}}}{\left[j_{\mathrm{RH}} j_{\mathrm{HR}}-\left(j_{\mathrm{RR}}-2 d_{\mathrm{R}}\right)\left(j_{\mathrm{HH}}-2 d_{\mathrm{H}}\right)\right]} .
$$

The term $j_{A B}$ represents the direct effect of species $B$ on species $A$ at the symmetric equilibrium. With this formula, we can note a few things about the possibility of the transition in the general model. First, as the no-dispersal equilibrium is stable only if $j_{\mathrm{RR}}<0$ and $\left|j_{\mathrm{RH}} j_{\mathrm{HR}}\right|>\left|j_{\mathrm{RR}} j_{\mathrm{HH}}\right|$ (app. A), when both $d_{\mathrm{R}}$ and $d_{\mathrm{H}}$ are equal to 0 , the level of predator dispersal required for the transition to spatially heterogeneous equilibria is negative, that is, cannot occur (app. A). Furthermore, increasing $d_{\mathrm{H}}$ such that $2 d_{\mathrm{H}}>j_{\mathrm{HH}}$ causes the critical level of predator dispersal rate to always be negative, no matter the dispersal rate of the basal resource. This will always hold if $j_{\mathrm{HH}}$ is negative, as negative herbivore dispersal rates cannot occur. However, if $d_{\mathrm{H}}$ is not very large and $j_{\mathrm{HH}}$ is positive, a sufficiently high resource dispersal rate can result in a positive critical predator dispersal rate. Therefore, this transition can occur only if the herbivore's growth rate responds positively to an increase in its own density at equilibrium. This requirement can be satisfied if the predator demonstrates saturation in its consumption of herbivores at equilibrium.

The critical dispersal level for the basal resource to transition to spatially symmetric, asynchronous oscillations is

$$
\begin{aligned}
d_{\mathrm{R}, \mathrm{c}}= & \frac{-B-\sqrt{B^{2}-4 A C}}{2 A}, \\
A= & -4\left(v_{\mathrm{HH}}+v_{\mathrm{PP}}\right), \\
B= & 4 j_{\mathrm{RR}}\left(v_{\mathrm{HH}}+v_{\mathrm{PP}}\right)-2\left(j_{\mathrm{RH}} j_{\mathrm{HR}}-v_{\mathrm{HH}}^{2}-2 v_{\mathrm{HH}} v_{\mathrm{PP}}-v_{\mathrm{PP}}^{2}\right), \\
C= & -j_{\mathrm{RR}}^{2}\left(v_{\mathrm{HH}}+v_{\mathrm{PP}}\right)+j_{\mathrm{RR}}\left(j_{\mathrm{RH}} j_{\mathrm{HR}}-v_{\mathrm{HH}}^{2}-2 v_{\mathrm{HH}} v_{\mathrm{PP}}-v_{\mathrm{PP}}^{2}\right) \\
& +v_{\mathrm{HH}}\left(j_{\mathrm{RH}} j_{\mathrm{HR}}+j_{\mathrm{HP}} j_{\mathrm{PH}}-v_{\mathrm{HH}} v_{\mathrm{PP}}\right)+v_{\mathrm{PP}}\left(j_{\mathrm{HP}} j_{\mathrm{PH}}-v_{\mathrm{HH}} v_{\mathrm{PP}}\right) .
\end{aligned}
$$

Here, $v_{\mathrm{HH}}=j_{\mathrm{HH}}-2 d_{\mathrm{H}}$ and $v_{\mathrm{PP}}=-2 d_{\mathrm{P}}$. What is imperative for a positive value for $d_{\mathrm{R}, \mathrm{c}}$ is that $A$ be negative, which can occur only if $j_{\mathrm{HH}}>2\left(d_{\mathrm{H}}+d_{\mathrm{P}}\right)$ (app. A). Once again, we need that $j_{\mathrm{HH}}$ be positive and that the dispersal of the herbivore be small relative to the herbivore's effect on itself at equilibrium. In addition, the dispersal of the predator also must be small for this transition to occur.

It is possible that either of these conditions could be satisfied in a system with hierarchical dispersal rates, depending on the type and strength of trophic interactions in the model. However, for any given system, both transitions are easier to achieve as dispersal rates become more nonhierarchical, 
either from increasing resource dispersal rates or reducing herbivore dispersal rates.

\section{Nonhierarchical Dispersal and Nonequilibrium Dynamics}

The effects of altering dispersal rates in nonequilibrium regimes may be stabilizing or destabilizing or may result in a complex shift in different stability criteria. As nonequilibrium dynamics are much less amenable to general analytical approaches, it is necessary to focus on a more concrete model. The remainder of our results are based on numerical and simulation analyses of the specific tritrophic model, equation (4).

While a six-dimensional system such as this can exhibit a wide variety of dynamics, the dynamics we observed in our system can be classified into five types (illustrated in fig. 1). These regimes can be classified according to their temporal dynamics and level of spatial heterogeneity. Temporally, the system can be in equilibrium or oscillating (including infiniteperiod chaotic oscillations). Spatially, the two patches can be dynamically homogeneous, asynchronous (both patches
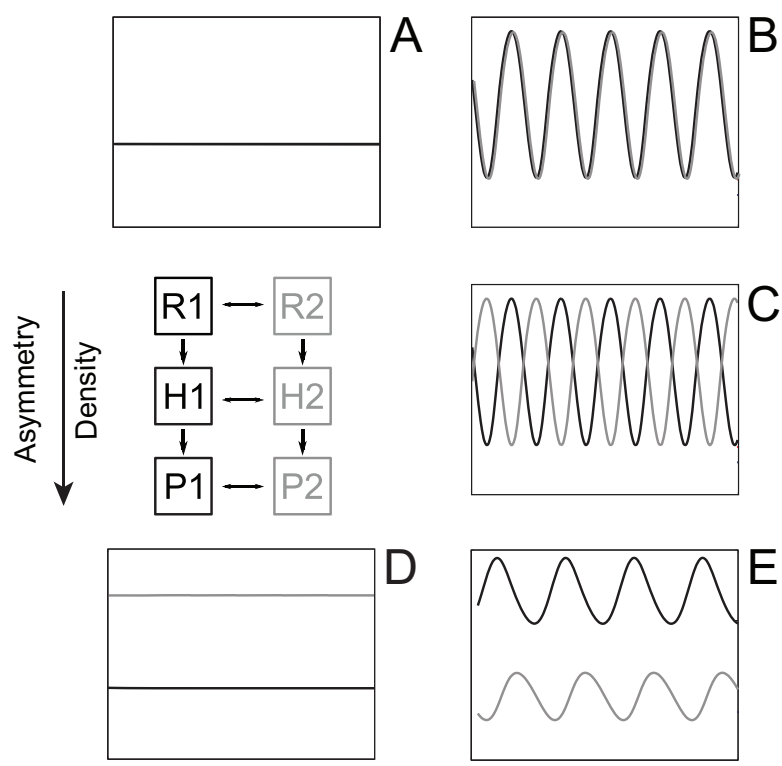

Time

Cyclicity

Figure 1: Possible spatiotemporal dynamics of the metacommunity. The colors (gray and black) denote which patch a population is in. Only the dynamics of the herbivore are shown in each figure, to simplify the presentation. The possible spatially homogeneous solutions are either an identical three-species equilibrium in both communities $(A)$ or perfectly synchronous oscillations $(B)$. The three possible spatially inhomogeneous solutions are a stable, asymmetric equilibrium for the two communities $(D)$, asynchronous oscillations, where both patches have the same range of densities but cycle in antiphase $(C)$, and asymmetric, out-of-phase oscillations $(E)$. exhibit the same dynamics but offset in time), or spatially asymmetric (the average levels for any of the resource, herbivore, or top consumer differ between sites).

Only five combinations of these are feasible (as an asynchronous equilibrium is not possible), all of which were found through bifurcation analysis in this system: homogeneous equilibrium (fig. $1 \mathrm{~A}$ ), homogeneous oscillations (fig. $1 B$ ), asynchronous oscillations (fig. 1C), asymmetric equilibrium (fig. $1 D$ ), and asymmetric oscillations (fig. $1 E$ ). In addition to the five types of attractor, bifurcation analysis revealed bistable parameter regions, where the system moved to one of two possible attractors, depending on initial conditions. We observed bistable symmetric oscillationsasymmetric equilibrium regions and bistable symmetric oscillations-asymmetric oscillations regions, although only the former ever formed a large enough region of parameter space to be observed through simulations. We found all of these states except asynchronous oscillation by varying the strength of self-regulation of the resource, $\beta$ (fig. 2 ), and setting dispersal rates at the boundary between nonmonotonic and antihierarchical dispersal structures $\left(d_{\mathrm{R}}=5, d_{\mathrm{H}}=0.1\right.$, and $d_{\mathrm{p}}=0.1$ ). In addition, we found that the symmetric limit cycles underwent a period-doubling bifurcation at low $\beta$ (labeled with an asterisk in fig. 2), indicating what may be the start of chaotic single-patch dynamics, previously observed to occur in this system (Hastings and Powell 1991). While our bifurcation analysis was not able to track the region of chaotic dynamics, our simulation analyses did not show any qualitative break in the stability of this system in response to variation in dispersal structure at $\beta$ values lower than the threshold where we observed the period-doubling bifurcation (fig. 3, dotted lines).

\section{Interacting Effects of Trophic and Dispersal Interaction Strength on Stability and Resilience}

The interacting effects of the vertical structure of dispersal rates and within-patch interaction rates can best be understood through pairwise bifurcation plots (figs. 3, 4). In general, trophic and dispersal interaction strengths interact to determine the stability and resilience of this system. In this model, variability is low and resilience is high when either $\beta$ is large or dispersal is strongly nonhierarchical. Further, the mechanism by which variability is reduced depends on whether dispersal is antihierarchical or nonmonotonic. The bifurcations between different dynamical regimes can be thought of as a set of predictions about what should be observed in a factorial experiment varying interactions and dispersal rates.

First, if herbivores disperse $\left(d_{\mathrm{H}}\right)$ more frequently than a minimum threshold, then the system will always approach a spatially homogeneous state, either an equilibrium or oscillations, depending on the value of $\beta$ (figs. $3 B, 4 A, 4 B$ ). 


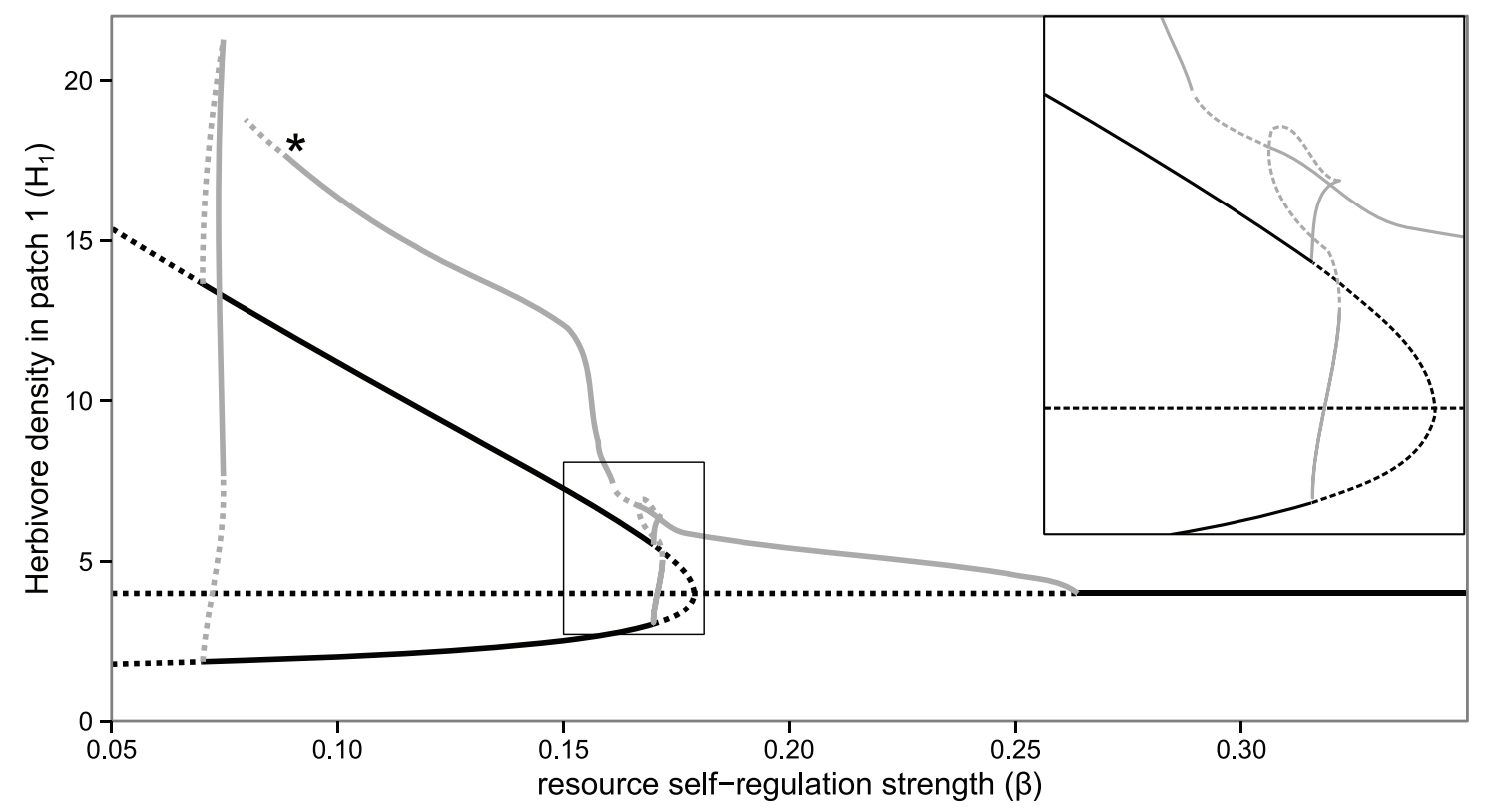

Figure 2: Bifurcation diagram showing changes in stability and maximum herbivore density of different solutions in one of the two patches as the strength of resource self-regulation $(\beta)$ changes. The lines represent where the maximum value of a given solution would lie for a given value of $\beta$. Solid lines are for stable solutions, and dashed lines indicate unstable solutions. Line color indicates what type of solution it is: black lines are for equilibria and gray lines are for limit cycles. For instance, when $\beta=0.1$, there are three solutions for the herbivore population in patch 1 : an unstable symmetric equilibrium at $H_{1} \approx 4.5$, an asymmetric equilibrium where $H_{1} \approx 2.5\left(H_{2} \approx 12\right)$ or $H_{1} \approx 12$ $\left(H_{2} \approx 2.5\right)$, or a symmetric limit cycle with $\max \left(H_{1}\right) \approx 17$. The point labeled with an asterisk marks a period-doubling bifurcation, which may indicate the onset of chaotic dynamics in a well-mixed system. The inset shows a magnified region near $\beta=0.15$, showing the complexity of the different solutions as $\beta$ varies in this range. Dispersal rates for this figure are set at $d_{\mathrm{R}}=5, d_{\mathrm{H}}=0.1$, and $d_{\mathrm{P}}=0.1$.

Second, the minimum level of $d_{\mathrm{H}}$ needed to observe spatially heterogeneous states varies strongly with the rate of resource self-limitation $(\beta)$. At both low and high levels of self-limitation, the range of possible dispersal rates that results in spatially inhomogeneous solutions is more limited (fig. 3).

Third, at intermediate levels of $\beta$, making dispersal more nonhierarchical by decreasing middle-trophic-level dispersal rates $\left(d_{\mathrm{H}}\right)$ or increasing both top- and bottom-trophic-level dispersal rates $\left(d_{\mathrm{R}}\right.$ and $\left.d_{\mathrm{P}}\right)$ leads to reduced variability because of the emergence of stable asymmetric equilibria (fig. 3).

Fourth, resource dispersal rates have to be at least an order of magnitude higher than herbivore dispersal rates to allow any spatially inhomogeneous solution to exist (fig. 4A).

Fifth, there are two alternate mechanisms by which dispersal structure can reduce aggregate variability (figs. $3 C, 4 B$, $4 C)$ : strongly antihierarchical dispersal $\left(d_{\mathrm{P}} \ll d_{\mathrm{H}}<d_{\mathrm{R}}\right)$ results in asymmetric equilibrium, and nonmonotonic or weakly antihierarchical dispersal $\left(d_{\mathrm{P}}>d_{\mathrm{H}}<d_{\mathrm{R}}\right)$ results in asynchronous oscillations. The transition between asynchronous and asymmetric dynamics does not always occur exactly at the boundary of nonmonotonic and antihierarchical dispersal rates. Instead, the transition depends on resource self-regulation (fig. $3 C$ ), resource dispersal rates (fig. $4 C$ ), and model structure (fig. B1; figs. B1, C1 available online).

Sixth, bistable symmetric oscillations-asymmetric equilibrium states appear when dispersal rates are at intermediate levels for all three trophic levels (fig. 3). At these levels, changing any dispersal rate can change the resilience of either asymmetric equilibrium or symmetric oscillations to perturbations, by resulting in the gain or loss of bistability. However, for the entire region of parameters and initial conditions explored, symmetric oscillations were substantially more likely than asymmetric equilibrium to occur, given that the system is in a bistable region. For a given parameter combination, which attractor the system ended up in was determined only by initial predator densities (fig. C1).

Seventh, asymmetric oscillations, while possible, did not occur for any significant range of parameters tested, except when both herbivore and predator dispersal $\left(d_{\mathrm{R}}\right.$ and $\left.d_{\mathrm{H}}\right)$ were very low (fig. $4 A$; but see app. B).

While the exact dispersal thresholds required to stabilize or destabilize a given tritrophic model will change under different assumptions about model structure, these broad results are robust under several different model structures (app. B). Further, we did not observe spatial stabilization 

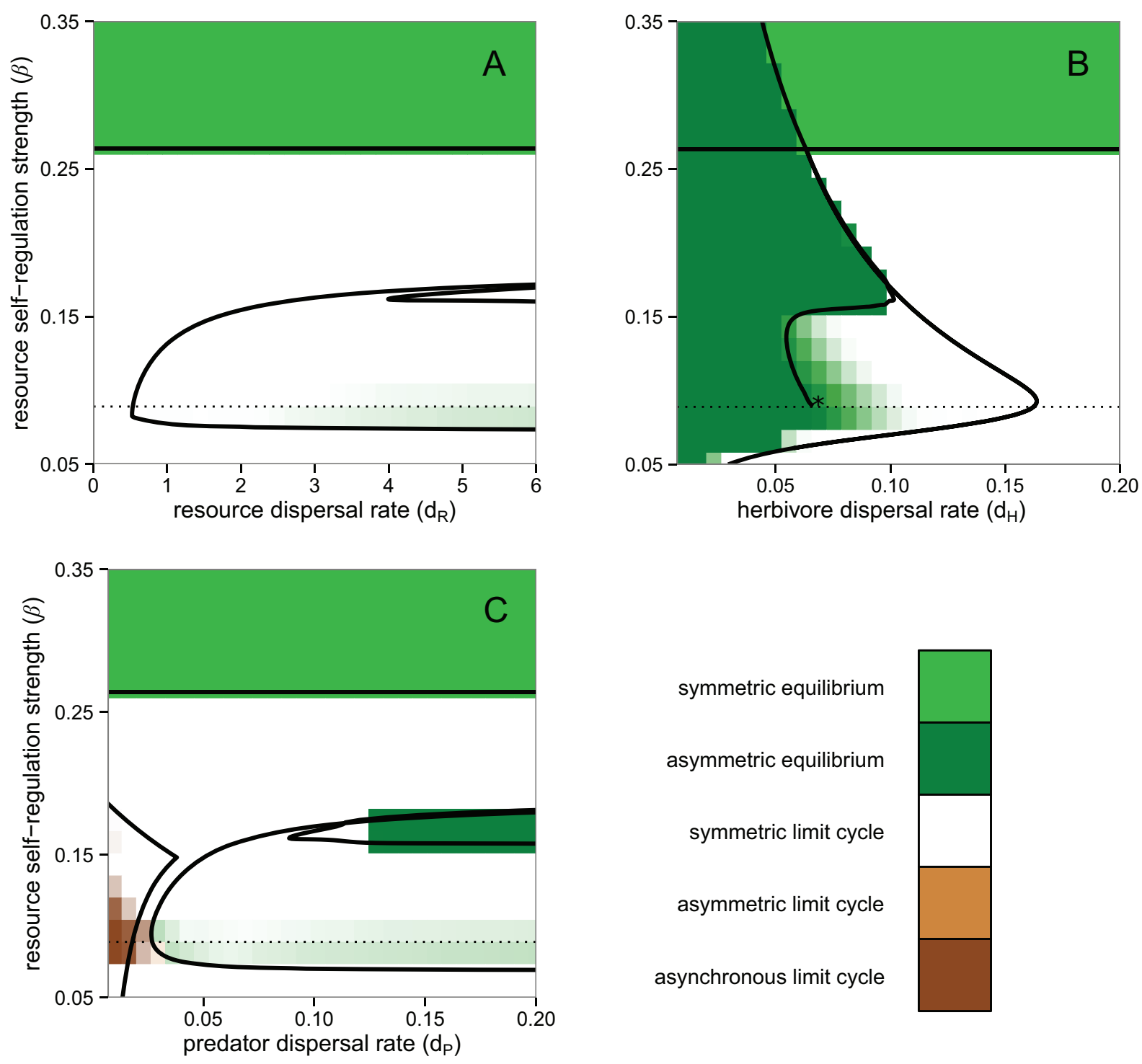

Figure 3: Changes in qualitative dynamics and relative volumes of different attractors from varying resource intraspecific interaction strength $(\beta)$ and dispersal rates, holding all other parameters constant. Lines indicate critical points separating regions with qualitatively different dynamics (only bifurcations leading to changes in qualitative dynamics are shown, for clarity). Grid points were colored according to the fraction of each type of dynamic observed. Each category of dynamic (fig. 1) was assigned a color. For grid cells where both symmetric oscillations (white) and another dynamic were observed (bistable dynamics), we colored the grid cell by taking the average RGB (red, green, blue) value of all the types of dynamics observed in the cell, weighted by how frequently each type was observed. This means that lowerintensity colors represent regions where oscillations are more prevalent than the alternate dynamic. The bifurcation line in $B$ that is marked with an asterisk does not end at that point. It was not possible to follow it numerically past there, however, because of the rapid increase in the period of oscillations. These rapid oscillations occurred near the point where the period-doubling bifurcation was observed in figure 1 (dotted lines). In any subplot where a given rate is not being varied, it is set to a base value: $d_{\mathrm{R}}=5, d_{\mathrm{H}}=0.1$, and $d_{\mathrm{P}}=0.1$.

with hierarchical dispersal for any parameter combination or alternate model parameterization (fig. B1).

\section{Discussion}

We have shown that stability increases under high resource and low herbivore dispersal. Further, both our analytical and numerical results show that the stabilizing mechanism depends on the dispersal rate of the top trophic level. Increasing the frequency of predator dispersal above a threshold leads to stability by forming stable spatial patterns. Decreasing predator dispersal below a threshold can stabilize global density fluctuations through the formation of outof-phase oscillations across space, leading to reduced mean 

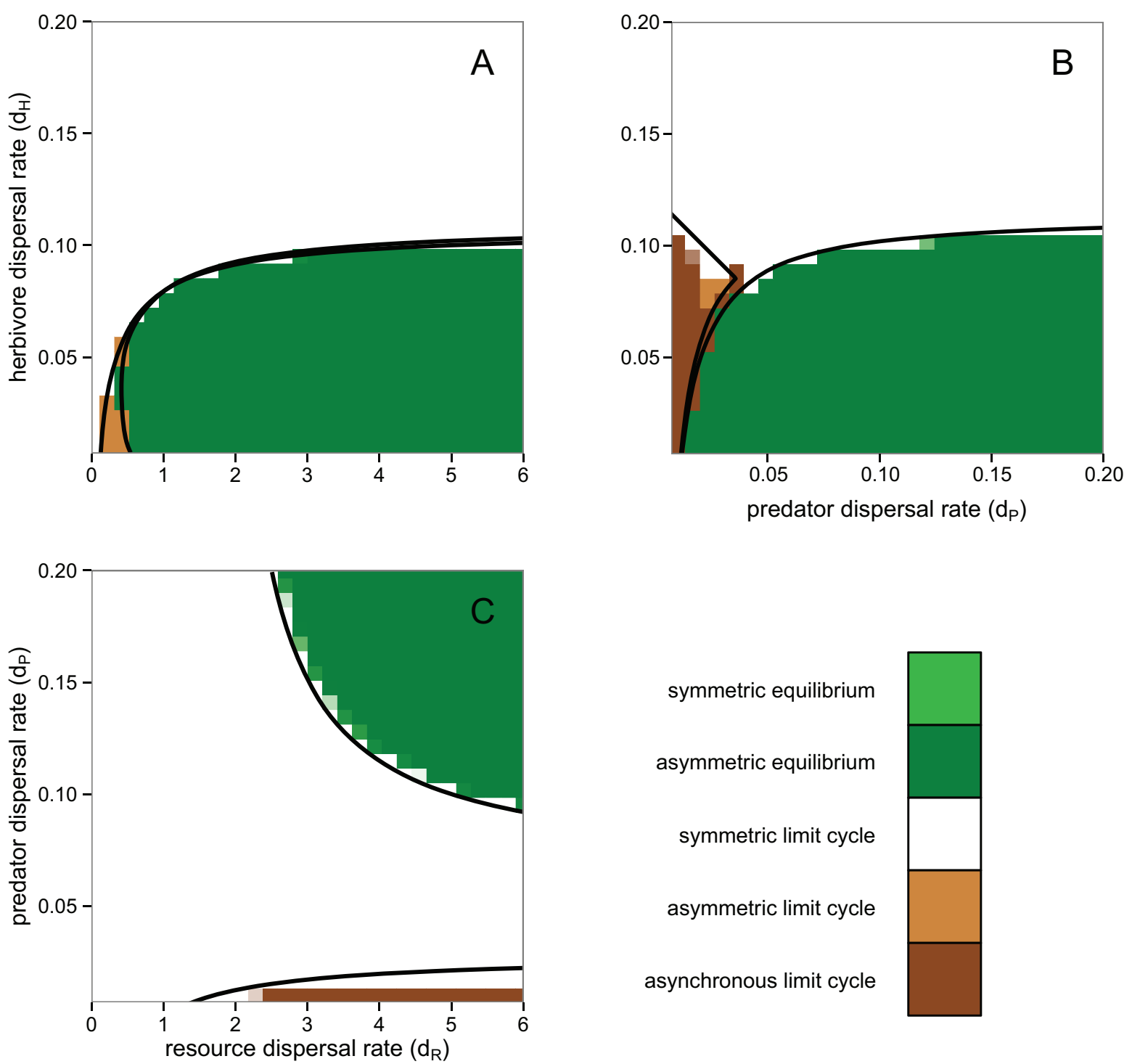

Figure 4: Changes in qualitative dynamics and relative volumes of different attractors from varying two dispersal rates simultaneously. Lines and colors are taken from continuation and simulation analyses as given in figure 3 . For these plots, $\beta=1 / 6$, and any subplot where a given rate is not being varied is set to a base value: $\beta=1 / 6, d_{\mathrm{R}}=5, d_{\mathrm{H}}=0.1$, and $d_{\mathrm{P}}=0.1$.

variability. We have also shown that increasing herbivore dispersal rates (fig. $3 B$ ) or decreasing predator dispersal rates (fig. $3 C$ ) may lead to a loss of ecological resilience, by shifting the system into a bistable state. For the model we used (and several alternate models; app. B), this meant that we observed spatial stabilization only when dispersal was nonhierarchical.

Nonhierarchical dispersal is not a necessary condition to destabilize tritrophic food webs. Previous work has shown that spatially inhomogeneous dynamics can occur in tritrophic systems where all species disperse at the same rate (e.g., Gouhier et al. 2010). What our analytical and numer- ical results do imply is that, all else being equal, a food web with equal or hierarchical dispersal will be more difficult to stabilize via pattern formation or asynchrony than one with nonhierarchical dispersal. Reducing herbivore dispersal or increasing resource dispersal should act to stabilize tritrophic food webs. Furthermore, as equations (5) and (6) indicate and our nonequilibrium results confirm, what matters is the combination of within-patch and between-patch interaction strengths (dispersal rates) for each species.

Our results formalize the treatment of dispersal as a type of ecological interaction and predict the relationship between the structure of dispersal across food chains and 
the spatiotemporal stability of metacommunities. Our study further stresses the importance of a common and explicit framework to integrate the topology of both trophic and spatial interactions.

\section{Dispersal as a Type of Interaction}

The concept of interaction strengths has been defined in a wide variety of ways in theoretical and empirical community ecology (Berlow et al. 2004). Here, we use one of the most common definitions: the interaction strength is the rate of change in species $A$ 's growth rate due to a change in the biomass of $B$ (May 1972). If we consider $A$ and $B$ to be two populations of the same species with constant per capita dispersal rates $d_{A B}$ and $d_{B A}$, respectively, then the interpopulation interaction strength of $B$ on $A$ will simply be $d_{A B}$. This is useful because it puts dispersal on an equal footing with other forms of ecological interactions. For instance, the conditions required for spatial pattern formation, that activators must disperse less frequently than inhibitors (Della Rossa et al. 2012), can be simply restated as follows: a species that interacts negatively with itself within a patch must have stronger interpatch interactions than a species with positive intraspecific interactions.

The flux of biomass between communities through dispersal can completely restructure the relative biomass of each species in the community and shift dynamical regimes. Trophic structure in dispersal rates lead to shifts in community stability, similar to the effect of trophic structure in interaction strengths within food webs (de Ruiter et al. 1995). The key structure is the relatively low dispersal rate of the herbivore relative to the predator or resource. That is, herbivore dispersal must be "weak" (infrequent) while dispersal at the other trophic levels must be "strong" (frequent) to affect stability.

Clearly, incorporation of dispersal as an interaction extends the reach of the interaction-strength framework for community stability as proposed by Rooney and McCann (2012). While some of the interaction-strength literature has considered spatial aspects of communities, it is limited to the assumption that spatial coupling occurs through interactions at higher trophic levels (Rooney et al. 2008). Here, we have shown that dispersal is a key interaction at all trophic levels and must be explicitly incorporated, especially if interpatch dispersal rates do not increase proportionately with increasing trophic level.

\section{What Movement Mechanisms Can Lead to Nonhierarchical Dispersal Structures?}

The bulk of studies that have included dispersal structure in food web dynamics have assumed that dispersal rates increase with trophic level. This assumption makes sense when dispersal over landscape scales for all species is driven by individuals moving between patches under their own power (McCann et al. 2005). However, many ecosystems are not simply chains of free-moving species.

Hierarchical dispersal may be violated in food chains with sedentary consumers. If the middle trophic level collects food by filtering out passing resources or by waiting in ambush for food, we would expect dispersal rates to appear nonmonotonic. The middle trophic level would disperse over the landscape less frequently than its resource, and mobile predators preying on the middle trophic level would disperse more frequently to capture their prey. For example, in the extensively studied Pisaster food web of the Pacific Coast, dispersal rates are nonmonotonic with respect to trophic position. There the intermediate trophic level is attached to the substrate. Mussels filter-feed on free-floating seston and are consumed by mobile predators such as Pisaster (Paine 1974), both of which disperse more frequently than mussels as measured in units of flux per unit biomass. Even when the long larval dispersal distances of Pisaster and mussels (Wieters et al. 2008) are taken into account, seston should disperse much more frequently, as it is constantly in the water column. Further, given the much longer pelagic larval duration of echinodermata relative to bivalves (Bradbury et al. 2008), Pisaster should disperse more frequently over generational timescales between distant sites than mussels. Paine (1966) identified other analogous food webs where the consumer dispersal rate is expected to be less frequent than that of the predator or the resource; however, this dispersal structure is expected generally where the consumer is attached to a substrate. In these cases, we would expect to see population dynamics stabilized by the emergence of fixed spatial patterns at the landscape scale.

Hierarchical dispersal may also break down when interpatch dispersal is driven by external transport mechanisms. For example, communities consisting of phytoplankton, zooplankton, and fish in small isolated or transient lakes will often be linked through animal-mediated dispersal, flooding, or wind dispersal of resting stages (Havel and Shurin 2004). All three of these mechanisms will tend to favor transporting smaller, lower-trophic-level species greater distances and in greater bulk (Beisner et al. 2006). This mechanism would result in an antihierarchical dispersal structure, which we predict would lead to stabilization through asynchrony between oscillating populations.

It is also important to recognize that, even in cases of independently moving organisms, large home-range size or maximum rate of movement over short timescales may not translate into high rates of dispersal between subpopulations. Behavioral decisions, driven by factors such as territoriality (Moorcroft and Lewis 2006) or fear-driven habitat avoidance, may prevent or reduce the chance of dispersal be- 
tween patches. For example, large predators, such as bears, may avoid road crossings when moving between forest patches (e.g., Mace et al. 1996). In this case, the degree to which the predator avoids long-distance dispersal between patches, rather than movement speed or home-range size, will determine how strongly populations of the predator interact.

Taken together, these examples show that hierarchical dispersal may not be the only structural pattern in food webs. The actual structure of dispersal rates will be determined by how different species in a food web answer the key conceptual questions of movement ecology: why move, how to get there, the timing and path to take, and how external factors influence movement (Nathan et al. 2008). We hope that this work will encourage more researchers to look for community-scale patterns in empirical dispersal data.

\section{Future Directions: Dispersal Structure in Complex Metacommunities}

Our results illustrate that nonhierarchical dispersal can increase stability and resilience in the simplest system that can be said to have structured dispersal: a tritrophic twopatch model. However, natural food webs will typically have more complex trophic structures and habitat connectivities. It remains unclear whether stability properties will be equally sensitive to trophic-level-specific dispersal rates in longer or more complex food webs.

One can argue that for longer food chains, all the intermediate trophic levels would have to disperse less frequently than both the top and bottom trophic levels, as any intermediate trophic level should exhibit dynamics similar to those of our "herbivore" level and thus act as an activator. This reinforces the idea that coastal benthic systems will be the most likely environments to detect trophically induced stabilization, being coupled through filter feeding of mobile plankton at the bottom and rapidly foraging fish at the top (as in Paine 1974; Menge et al. 1997). For instance, Della Rossa et al. (2013) showed that in nutrient-phytoplankton-zooplanktonfish models, infrequent zooplankton dispersal is necessary to form spatial patterns, but infrequent phytoplankton dispersal may enhance or inhibit spatial patterns, depending on the underlying trophic interaction strengths.

Our two-patch formulation also ignored the fact that species not only may differ in average dispersal rates between patches but also will generally disperse over the landscape in different ways. For example, smaller species can gain access to finer-scale resources and refuges inaccessible to larger species (Ritchie 2009), high-risk prey may avoid moving through open areas between patches, and lighter plankton may undergo more frequent long-distance transmission than heavier species. Recent multiscale theoretical approaches may be more useful than the metacommunity approach for understanding dispersal variability that occurs across multiple scales, by modeling dispersal as nonlinear lagged terms (Keeling et al. 2000) or global modifications to local functional responses (Barraquand and Murrell 2013) or through explicit terms modeling spatial variability (Ovaskainen et al. 2014). The question here, however, is the same as for more complex food webs: what combination of species interactions and dispersal structures that are observed in nature can increase stability?

\section{Acknowledgments}

We gratefully acknowledge funding from the Centre for Applied Mathematics in Bioscience and Medicine, the National Institute for Mathematical and Biological Synthesis, and the Mathematical Biosciences Institute to attend the Joint 2011 Summer Graduate Workshop in Mathematical Ecology and Evolution. E.J.P. and M.G. acknowledge funding from Quebec Ocean. E.J.P. and F.G. also acknowledge Natural Sciences and Engineering Research Council funding. J.N.M. acknowledges funding from the Quebec Center for Biodiversity Science and Fonds Québécois de la Recherche sur la Nature et les Technologies, and H.V.M. acknowledges a National Science Foundation Graduate Research Fellowship. E.J.P. thanks E. J. Doedel for advice and training on continuation analysis. We also thank S. Connolly, D. Green, E. LowDecarie, A. Rossberg, P. Thompson, C. Ziter, and two anonymous reviewers for their feedback, which improved this work substantially. All simulations were run on the Guillimin server from McGill University, managed by Calcul Québec and Compute Canada. The code used for all simulations is available at dx.doi.org/10.5281/zenodo.31832.

\section{Literature Cited}

Allesina, S., and S. Tang. 2012. Stability criteria for complex ecosystems. Nature 483:205-208.

Alonso, D., F. Bartumeus, and J. Catalan. 2002. Mutual interference between predators can give rise to Turing spatial patterns. Ecology 83:28-34.

Barraquand, F., and D. J. Murrell. 2013. Scaling up predator-prey dynamics using spatial moment equations. Methods in Ecology and Evolution 4:276-289.

Beisner, B. E., P. R. Peres-Neto, E. S. Lindström, A. Barnett, and M. L. Longhi. 2006. The role of environmental and spatial processes in structuring lake communities from bacteria to fish. Ecology 87: 2985-2991.

Berlow, E. L., A.-M. Neutel, J. E. Cohen, P. C. de Ruiter, B. Ebenman, M. Emmerson, J. W. Fox, et al. 2004. Interaction strengths in food webs: issues and opportunities. Journal of Animal Ecology 73:585-598.

Bradbury, I. R., B. Laurel, P. V. Snelgrove, P. Bentzen, and S. E. Campana. 2008. Global patterns in marine dispersal estimates: the influence of geography, taxonomic category and life history. Proceedings of the Royal Society B: Biological Sciences 275:1803-1809. Carnell, R. 2012. lhs: Latin hypercube samples. R package version 0.10. https://cran.r-project.org/web/packages/lhs/index.html. 
Della Rossa, F., S. Fasani, and S. Rinaldi. 2012. Potential Turing instability and application to plant-insect models. Mathematical and Computer Modelling 55:1562-1569.

2013. Conditions for patchiness in plankton models. Theoretical Population Biology 83:95-100.

de Ruiter, P. C., A.-M. Neutel, and J. C. Moore. 1995. Energetics, patterns of interaction strengths, and stability in real ecosystems. Science 269:1257-1260.

Doedel, E. J., A. R. Champneys, T. F. Fairgrieve, Y. A. Kuznetsov, B. Sandstede, and X. Wang. 2010. AUTO-07p: continuation and bifurcation software for ordinary differential equations. http://indy .cs.concordia.ca/auto.

Fagan, W. F. 2002. Connectivity, fragmentation, and extinction risk in dendritic metapopulations. Ecology 83:3243-3249.

Fasani, S., and S. Rinaldi. 2011. Factors promoting or inhibiting Turing instability in spatially extended prey-predator systems. Ecological Modelling 222:3449-3452.

Gouhier, T. C., F. Guichard, and B. A. Menge. 2010. Ecological processes can synchronize marine population dynamics over continental scales. Proceedings of the National Academv of Sciences of the USA 107:8281-8286.

Guichard, F., and T. C. Gouhier. 2014. Non-equilibrium spatial dynamics of ecosystems. Mathematical Biosciences 255:1-10.

Gunderson, L. H. 2000. Ecological resilience: in theory and application. Annual Review of Ecology and Systematics 31:425-439.

Haskell, J. P., M. E. Ritchie, and H. Olff. 2002. Fractal geometry predicts varying body size scaling relationships for mammal and bird home ranges. Nature 418:527-530.

Hastings, A., and T. Powell. 1991. Chaos in a three-species food chain. Ecology 72:896-903.

Havel, J. E., and J. B. Shurin. 2004. Mechanisms, effects, and scales of dispersal in freshwater zooplankton. Limnology and Oceanography 49:1229-1238.

Ims, R. A., J.-A. Henden, and S. T. Killengreen. 2008. Collapsing population cycles. Trends in Ecology and Evolution 23:79-86.

Jansen, V. A., and A. L. Lloyd. 2000. Local stability analysis of spatially homogeneous solutions of multi-patch systems. Journal of Mathematical Biology 41:232-252.

Justus, J. 2006. Loop analysis and qualitative modeling: limitations and merits. Biology and Philosophy 21:647-666.

Keeling, M. J., H. B. Wilson, and S. W. Pacala. 2000. Reinterpreting space, time lags, and functional responses in ecological models. $\underline{\text { Sci- }}$ ence 290:1758-1761.

Kendall, B. E., J. Prendergast, and O. N. Bjørnstad. 1998. The macroecology of population dynamics: taxonomic and biogeographic patterns in population cycles. Ecology Letters 1:160-164.

Koelle, K., and J. Vandermeer. 2005. Dispersal-induced desynchronization: from metapopulations to metacommunities. Ecology Letters 8:167-175.

Levins, R. 1974. The qualitative analysis of partially specified systems. Annals of the New York Academv of Sciences 231:123-138

Loreau, M., A. Downing, M. Emmerson, A. Gonzalez, J. Hughes, P. Inchausti, J. Joshi, J. Norberg, and O. Sala. 2002. A new look at the relationship between diversity and stability. Pages 79-91 in M. Loreau, S. Naeem, and P. Inchausti, eds. Biodiversity and ecosystem functioning: synthesis and perspectives. Oxford University Press, Oxford.

Mace, R. D., J. S. Waller, T. L. Manley, L. J. Lyon, and H. Zuuring. 1996. Relationships among grizzly bears, roads and habitat in the
Swan Mountains, Montana. Journal of Applied Ecology 33:13951404.

Marleau, J. N., F. Guichard, and M. Loreau. 2014. Meta-ecosystem dynamics and functioning on finite spatial networks. Proceedings of the Roval Societv B: Biological Sciences 281:20132094. doi:10 $.1098 / \mathrm{rspb} .2013 .2094$.

Marleau, J. N., F. Guichard, F. Mallard, and M. Loreau. 2010. Nutrient flows between ecosystems can destabilize simple food chains. Lournal of Theoretical Biology 266:162-174.

May, R. M. 1972. Will a large complex system be stable? Nature 238:413-414.

- 1973. Stability and complexity in model ecosystems. Princeton University Press, Princeton, NJ.

McCann, K. S., A. Hastings, and G. R. Huxel. 1998. Weak trophic interactions and the balance of nature. Nature 395:794-798.

McCann, K. S., J. B. Rasmussen, and J. Umbanhowar. 2005. The dynamics of spatially coupled food webs. Ecology Letters 8:513-523.

Menck, P. J., J. Heitzig, N. Marwan, and J. Kurths. 2013. How basin stability complements the linear-stability paradigm. Nature Physics 9:89-92.

Menge, B. A., B. A. Daley, P. A. Wheeler, E. Dahlhoff, E. Sanford, and P. T. Strub. 1997. Benthic-pelagic links and rocky intertidal communities: bottom-up effects on top-down control? Proceedings of the National Academy of Sciences of the USA 94:14530-14535.

Moorcroft, P. R., and M. A. Lewis. 2006. Mechanistic home range analysis. Princeton University Press, Princeton, NJ.

Nathan, R., W. M. Getz, E. Revilla, M. Holyoak, R. Kadmon, D. Saltz, and P. E. Smouse. 2008. A movement ecology paradigm for unifying organismal movement research. Proceedings of the National Academy of Sciences of the USA 105:19052-19059.

Neutel, A.-M., J. A. P. Heesterbeek, and P. C. de Ruiter. 2002. Stability in real food webs: weak links in long loops. Science 296:1120-1123.

Ovaskainen, O., D. Finkelshtein, O. Kutoviy, S. Cornell, B. Bolker, and Y. Kondratiev. 2014. A general mathematical framework for the analysis of spatiotemporal point processes. Theoretical Ecology 7:101-113.

Paine, R. T. 1966. Food web complexity and species diversity. American Naturalist 100:65-75.

. 1974. Intertidal community structure: experimental studies on the relationship between a dominant competitor and its principal predator. Oecologia (Berlin) 15:93-120.

-1992. Food-web analysis through field measurement of per capita interaction strength. Nature 355:73-75.

Pascual, M. 1993. Diffusion-induced chaos in a spatial predator-prey system. Proceedings of the Roval Society B: Biological Sciences 251:1-7.

Pillai, P., A. Gonzalez, and M. Loreau. 2011. Metacommunity theory explains the emergence of food web complexity. Proceedings of the National Academv of Sciences of the USA 108:19293-19298.

R Core Team. 2013. R: a language and environment for statistical computing. R Foundation for Statistical Computing, Vienna.

Rietkerk, M., and J. van de Koppel. 2008. Regular pattern formation in real ecosystems. Trends in Ecology and Evolution 23:169-175.

Ritchie, M. E. 2009. Scale, heterogeneity, and the structure and diversity of ecological communities. Princeton University Press, Princeton, NJ.

Rooney, N., and K. S. McCann. 2012. Integrating food web diversity, structure and stability. Trends in Ecology and Evolution 27:40-46.

Rooney, N., K. S. McCann, and J. C. Moore. 2008. A landscape theory for food web architecture. Ecology Letters 11:867-881. 
Segel, L. A., and J. L. Jackson. 1972. Dissipative structure: an explanation and an ecological example. Journal of Theoretical Biology 37:545-559.

Soetaert, K., T. Petzoldt, and R. W. Setzer. 2010. Solving differential equations in R: package deSolve. Journal of Statistical Software 33:1-25.

Turing, A. M. 1952. The chemical theory of morphogenesis. Philosophical Transactions of the Roval Societv B: Biological Sciences 237:32.

van Voorn, G. A., B. W. Kooi, and M. P. Boer. 2010. Ecological consequences of global bifurcations in some food chain models. Mathematical Biosciences 226:120-133.
Wieters, E., S. Gaines, S. Navarrete, C. A. Blanchette, and B. Menge. 2008. Scales of dispersal and the biogeography of marine predatorprey interactions. American Naturalist 171:405-417.

Wootton, J. T. 1997. Estimates and tests of per capita interaction strength: diet, abundance, and impact of intertidally foraging birds. Ecological Monographs 67:45-64.

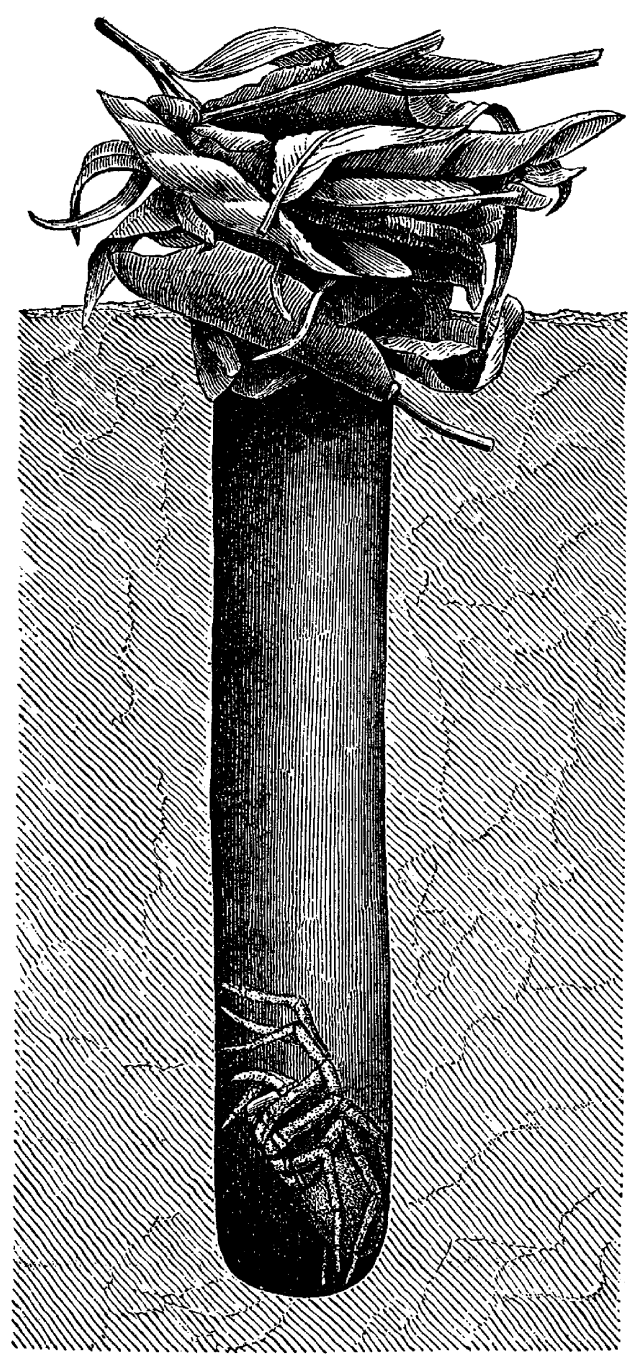

"The holes were from six to eight inches deep and lined with a delicate web, which near the top was stout enough to be separated from the sand, forming a silken tube attached to the ring of chips around the mouth of the hole.... Before opening the holes we sounded them with straws and tried to provoke the spiders to come out, but they took no notice of it." From "The Lycosa at Home" by J. H. Emerton (The American Naturalist, 1871, 4:664-665). 